\title{
Therapeutic Basis of Human Diseases Based on Metabolome-related Knowledge
}

\author{
Khaled Mohamed Mohamed Koriem ${ }^{1, * i}$ \\ 1 Department of Medical Physiology, Medical Research Division, National Research Centre, 33 El-Buhouth Street, Dokki, \\ Cairo, 12622, Egypt \\ * Correspondence: kkoriem@yahoo.com;
}

Scopus Author ID 24477156100

Received: 25.02.2021; Revised: 30.03.2021; Accepted: 3.04.2021; Published: 20.04.2021

\begin{abstract}
The metabolome is mentioned to small molecules inside the biological sample. The metabolome includes endogenous metabolites such as natural compounds such as nucleic acids, amino acids, vitamins, fatty acids, sugars, and exogenous compounds such as food additives, environmental toxicants, drugs xenobiotics. Human disease is induced by external factors e.g., pathogens or internal dysfunctions e.g: hypersensitivity, immunity disorders, autoimmune disorders, immunodeficiency, and allergy. There are 6 different types of human diseases: human acquired disease, human acute disease, human chronic disease, human congenital disease, human genetic disease, and incurable human disease. Human acute disease is appears suddenly and persists for a short human time. Human acute diseases such as influenza virus and the disease symptoms include fever. Human chronic diseases remain for 1 year or more and need continuing medical precaution or limit activities of human dailydaily human life activities. Human chronic diseases include heart diseases, cancers, and diabetes that cause human death or disability. Human congenital disease can be developed in the fetal growth stage or from the parents' genetic transfer. Genetic disease is a human health problem induced via one or more defects in the genome. The genetic disease is induced via one or more defects in the genome. In conclusion, the metabolome plays an important role in the therapeutic basis of all the above mentioned human diseases.
\end{abstract}

Keywords: metabolome; human; acute; chronic; genetic; disease; therapy.

(C) 2021 by the authors. This article is an open-access article distributed under the terms and conditions of the Creative Commons Attribution (CC BY) license (https://creativecommons.org/licenses/by/4.0/).

\section{Introduction}

The metabolome is mentioned to small molecules inside the biological sample [1]. The metabolome includes both endogenous metabolites such as natural compounds secreted by an organism, e.g., nucleic acids, amino acids, vitamins, fatty acids, sugars, antibiotics, etc. The metabolome also contains exogenous compounds e.g., food additives, environmental toxicants, drugs, and xenobiotics. So, the metabolome includes both an endogenous metabolome and an exogenous metabolome. The endogenous metabolome is divided into primary and secondary metabolome. The primary metabolite includes normal reproduction, growth, and development. The secondary metabolite includes antibiotics and pigments. The research of the metabolome is termed metabolomics [2,3]. The metabolome refers to metabolite and chromosome i.e., metabolites are controlled by genes or act on genes. The metabolome was first appeared in 1998 [1, 4]. The metabolome is the $4^{\text {th }}$ generation following genome, proteome, and transcriptome. There is a connection between lipidome, proteome, and metabolome [5-8]. The metabolome is a connection between the genome and the environment. Nuclear magnetic resonance (NMR) and mass spectrometry (MS) are the famous techniques used in the 
metabolome. The human metabolome database (HMDB) is an available, open-access database of human metabolites that possesses higher than 40,000 metabolites identified inside the human body. The HMDB contains chemical, clinical, and biochemical data. The chemical data contain higher than 40,000 metabolite structures. The clinical data contains 10,000 metabolite-biofluid concentrations. The biochemical data contains 6000 protein structures and 5000 biochemical reactions.

Diseases are medical cases correlated with definite symptoms and syndromes. Humans' disease is accompanied by distress, pain, social problems, dysfunction, or death. There are 6 different types of human diseases: human-acquired disease, human acute disease, human chronic disease, human congenital disease, human genetic disease, and incurable human disease. The acquired disease is a disease that is initiated at any time of the human lifetime. This disease is different from another disease that occurred at human birth (congenital disease). Human acute disease is appears suddenly and persists for a short human time. This disease is varied from chronic diseases (which grow slowly and sustains for many human months or until the human end of life). Human acute diseases such as influenza virus and the disease symptoms include fever. Human chronic diseases are remained for 1 year or more and need continuing medical precaution or limit activities of daily human life. Human chronic diseases include heart diseases, cancers, and diabetes that cause human death or disability. The human congenital disease is a medical state that is occurred at or before human birth. This disease can be developed in the fetal growth stage or from the genetic transfer from the parents. Genetic disease is a human health problem induced via one or more defects in the genome. This disease is induced by a mutation through a single gene (monogenic) or many genes (polygenic) or by a chromosomal aberration.

This review aims to focus on the therapeutic basis of human diseases based on metabolome-related knowledge.

\section{Metabolome}

The metabolome includes both endogenous metabolites such as natural compounds secreted by an organism e.g., nucleic acids, amino acids, vitamins, fatty acids, sugars, antibiotics, etc. The metabolome also contains exogenous compounds e.g., food additives, environmental toxicants, drugs, and xenobiotics. So, the metabolome includes both an endogenous metabolome and an exogenous metabolome. The endogenous metabolome is divided into primary and secondary metabolome. The primary metabolite includes normal reproduction, growth, and development. Metabolomics studies show that not all metabolites are created equally in aging biology. The hub metabolites are created as central mediators of aging. The hub metabolites are $\alpha$-ketoglutarate, nicotinamide dinucleotide phosphate, nicotinamide adenine dinucleotide, and $\beta$-hydroxybutyrate. These hub metabolites include cofactors or intermediates of carbon metabolism [9]. Metabolome study provides a good detection of more than 800 metabolites, flagging the way towards understanding variations in metabolites in different conditions and designing involvements for controlling the levels of circulating metabolites [10]. In the microbial process, a human gastrointestinal tract is a place to an extremely microbes. These microbes capture energy in an anaerobic condition. The breakdown of dietary stuff and gut microbiota produces many metabolic products that accumulate to high amounts in the gut. These chemicals influence host biology in 2 ways; (1) by an act on cells within the gastrointestinal tract and (2) enter the blood circulation and exert their effects within the body [11]. The bacteria are associated with a decline of 50 human proteins and free amino 
acids while increasing bacterial fermentation such as acetate and succinate. The fermentation of amino acids provides a mechanism for the initial growth of Escherichia coli in an anaerobic condition [12]. Metabolome analysis reveals a tissue-specific control of metabolism. Metabolome detects correlation of both central and peripheral metabolites with Alzheimer's disease. There is little connection between blood and brain metabolome in Alzheimer's disease [13]. Metabolome study reveals that leptin is correlated with lipolysis and substrate utilization, which leads to negative energy balance. The leptin's effects on branched-chain amino acids and steroid metabolites and consequently lead to decline caloric consumption [14]. Circadian disorder is associated with metabolic disturbance such as energy or redox imbalances. Circadian disorder is associated with sleep loss. The metabolic significances are explored by metabolomic analysis of circadian biology and sleep [15]. Blood metabolome analysis is used in the medical field. In metabolome study, the blood samples from healthy persons rise to a healthy person's metabolomic image. It allows researchers to draw a map for human blood metabolome with the normal state. A $99 \%$ of the metabolic processes that occur in the blood plasma are evaluated. In an abnormal state, the metabolomic image allows getting the value of these deviations of metabolic processes [16]. The gut microbiome is one of the major players in the metabolome. The changes in metabolite state due to variations in the gut microbiome occur due to a cause or consequence of renal diseases. The renal-metabolome-microbiome link has a major role in the diagnosis and control of renal diseases [17]. Metabolome study is very important in detecting children with food allergy which occurs in the presence of asthma. There is a decline in children with food allergies in ceramides and sphingolipids levels, and these observations reflect the metabolomic alterations. These observations mentioned the connection between an altered microbiota and immunity in the gut [18]. Metabolome, transcriptome, and proteome play an important role in the connection beween pathogen and the host in human diseases. The infection diseases include many biological processes such as variation of the host immunity, homeostasis, and host's metabolic process with the microbiome [19]. Metabolome analysis provides a good tool for the Mulberry plant, which has used in traditional medicine. The results reveal that this plant contains 124 metabolites, and their chemical structures have been detected and deposited in the database [20]. Metabolome study is used to explore plant metabolites such as glucosinolates, carotenoids, and terpenoids. Metabolome data helps the researchers understand the importance of cell constituent detection of metabolite-protein complexes [21]. Metabolome study help to understand the therapeutic effect of Bushen Tiansui Formula (is a traditional Chinese medicine to treat Alzheimer's disease). Metabolome identifies the active components and mechanisms related to this traditional Chinese's pharmacological effects [22]. Metabolome analysis identifies early-stage biomarkers in Alzheimer's disease and age-related cognitive decline to develop preventative treatments [23]. Metabolome study in soybean helps to understand the response of soybean to different stresses such as heat, flooding, drought, salinity, chilling, and heavy metal stresses. Also, the metabolites detected in response to different stresses provide a reference for metabolomics applications in soybean research [24].

\section{Role of Metabolome in Human Diseases}

Human disease is an abnormal case that negatively affects the structure and function of one organism of the whole human body. Diseases are medical cases correlated with definite symptoms and syndromes. Human disease is induced by external factors e.g., pathogens, or by internal dysfunctions, e.g., hypersensitivity, immunity disorders, autoimmune disorders, 
immunodeficiency, and allergy. Humans' disease is accompanied by distress, pain, social problems, dysfunction, or death. So, human diseases are associated with disabilities, syndromes, injuries, disorders, infections. Human diseases are physical or mental. There are 6 different types of human diseases such as; acquired disease (begins at any time of human life), acute disease (is a short-time disease), chronic disease (long time disease e.g. six months or whole human life), congenital disease (diseases occurs at birth), genetic disease (induced by one or more genetic mutations), incurable disease (disease without any treatment). The metabolome represents a new and accurate method to detect ovarian cancer in the early stage. Metabolome is applied through using serum, urine, tissue, plasma, ascites, or exosomes. The early diagnosis of ovarian cancer has a potential role in the disease curing. The metabolites and metabolism possess an important role in both cancer biology and biomarkers discovery [25]. Metabolome affects the pro-inflammatory reaction of microglia, and this reaction explain the mechanism of many neurodegenerative diseases. The toll-like receptor-4 or triggering receptors expressed on myeloid cells- 2 and pro-inflammatory signaling paths initiate the caspase-associated immune reaction of microglia [26]. The metabolomic analysis is a biomarker of bronchopulmonary dysplasia and other neonatal diseases, which explores the effect of acylcarnitine, amino-acids, and fatty acids in lung damage and healing. These nutrients, such as lipids, amino acids, and vitamins, possess an effect on lung structure and function and consequently affect acute and chronic respiratory disturbance development. Human milk inhibits bronchopulmonary dysplasia in children through inflammation variation and repairs antibiotic therapy-facilitated microbiome disturbance [27]. Metabolome analysis of stool obtained from ileum examines the role of primary bile acids in intestinal inflammation. The mechanism includes decline deoxycholic acid and lithocholic acid levels, and genes transform primary bile acids to secondary bile acids. Secondary bile acids decline intestinal inflammation [28]. Metabolome analysis is very important to detect the metabolic profile of the brain in Parkinson's disease. The dopamine content of the brain, myo-inositol, dehydroascorbic acid, and glycine are declined, while threonine is increased in the mesencephalon area of the brain of Parkinson's disease. In Parkinson's disease, mannose and fructose are increased while urea and glycine are decreased [29]. Metabolomics analysis explores the mechanisms of autoimmune diseases and identifies new therapeutic agents for these diseases. The metabolome gives more details on the concept of "skilled immunity" to increase autoimmunity and autoinflammatory treatments [30]. Metabolome analysis is a suitable method to detect the mechanism involved in breast cancer. The mitochondrial serine pathway pushes the faster multiplying of breast cancer through increases in purine synthesis. In human breast cancer, the expression of genes in the mitochondrial serine is correlated with disease consequences. This case also occurs in adrenocortical carcinoma and kidney chromophobe cell carcinoma. So, mitochondrial serine possesses an important role in increasing cancer development [31]. Metabolome analysis in breast milk analysis discovers its metabolic pathways. This enables researchers to investigate its effect on newborn growth. The metabolome is also useful to identify breast milk-associated stem cell markers, possessions, and differences in different lactation stages, to explore milk's role in the healing field [32]. Metabolomic analysis of gut microbiota is used in 11 patients with non-small cell lung cancer. The results reveal 4/11 patients possess early development, while $7 / 11$ possess cancer development after 12 months. The tridecane and 2-Pentanone metabolites components are correlated with early cancer, while short-chain fatty acids such as butyrate, propionate, lysine, and nicotinic acid are correlated with useful properties [33]. The metabolome is a suitable method to investigate the effect of 
Dichlorodiphenyltrichloroethane (DDT) on human health. There are many changes in human metabolites due to DDT exposure, e.g., amino acids such as arginine possess a strong correlation with DDT exposure in humans and animals. Also, lipids and acyl-carnitine intermediates strongly correlate with DDT exposure in humans which refers to mitochondrial damage. Consequently, serine and fatty acid metabolism have a vital role in DDT disease initiation [34]. The human diseases include acquired disease, acute disease, chronic disease, congenital disease, genetic disease, and incurable disease as follows:-

\subsection{Role of the metabolome in human acquired disease.}

The acquired disease is a disease that is initiated at any time of the human lifetime. This disease is different from another disease that occurred at human birth (congenital disease). The acquired disease is means acquired disease after birth. Metabolome analysis is a suitable method to investigate the gene expression mechanism in Alzheimer's disease, such as trigger receptor expressed inside myeloid cells-2 (TREM-2) in the human brain. The TREM-2 is an immunity receptor expressed in microglia and increases Alzheimer's disease initiation. The TREM2-deficient microglia myelin occurs in Alzheimer's disease and leads to cholesteryl ester increase and diminishes brain cholesterol transport [35]. The metabolome used in the detection of hepatocellular carcinoma. The hepatitis $\mathrm{C}$ and $\mathrm{B}$ viral infections are the main threat factors for hepatocellular carcinoma that causes chronic liver disease. The hepatocellular carcinoma induces chemical changes of 194 compounds' structure. The most changed compounds are the class of sugar alcohols. In hepatocellular carcinoma tissues, sugar alcohols were the most differentiating compounds in hepatocellular carcinoma tissues. The change of sugar alcohol in liver tissues detects in the early stage of hepatocellular carcinoma. So, the increase in sugar alcohol level refers to the vital role of aldo-keto reductases in hepatocellular carcinoma [36]. Metabolome analysis reveals that alveolar macrophages improved from pneumonia have new activities and changed responses to infection. The acquired lung protection following minor bacterial respiratory infections includes changes of remodeling of the alveolar macrophage to be long-lasting infection resistance [37]. Metabolome analysis explores the change in opioid receptors due to morphine exposure, leading to disturbance in gastrointestinal tract barrier function and, consequently, increases the translocation of gastrointestinal tract commensal bacteria. This study established a positive correlation between opioid drug use/abuse and an increased threat of infections and leads to an increase in the susceptibility to hospital-acquired infection [38]. The cytokine causes changes in metabolome factors. This concept helps in the treatment of inflammatory and autoimmunity diseases. The Thelper 17 cells are very important in autoimmunity and cancer. The metabolome is very important to develop and observe the new biomarkers. The biomarkers possess a vital role in treating patients with cancer, i.e., early discovery of the disease, threat stratification, diagnostic precision, treatment, and disease follow-up. The biomarkers are divided into 4 groups: (1) cytogenetic abnormalities, (2) protein antigens, (3) gene expression, and (4) genetic polymorphisms [40]. Metabolome analysis plays an important role in investigating the sulfur role in protein structure and function and redox state and signaling in humans. Human physiology and pathophysiology depend on hydrogen sulfide $\left(\mathrm{H}_{2} \mathrm{~S}\right)$ and sulfane sulfur (SS). Human diseases are correlated with abnormal levels of $\mathrm{H}_{2} \mathrm{~S} / \mathrm{SS}$ in blood and tissues. The treatment by $\mathrm{H}_{2} \mathrm{~S}$ intake is used for disease avoidance and as anti-aging factors [41]. Metabolome analysis gives more detail about human microbiota and their roles in the homeostasis of human health. In acquired human disease, the microbial genes structures and functions are changed to sustain human health and diseases [42]. Metabolome 
analysis is very important in human health and disease such as asthma. Metabolome research includes sample collection, analysis, and reading of asthma patients [43]. Metabolome analysis occurs in human disease such as high cholesterol level is correlated with the many diseases such as neurodegenerative and metabolic disturbances, cardiovascular diseases, and tumors. The oxidation of cholesterol to oxysterols occurs in human disease. Oxysterols play an important role in the diagnosis and treatment of human diseases [44].

\subsection{Role of the metabolome in human acute disease.}

Human acute disease is appears suddenly and persists for a short human time. This disease is varied from chronic diseases (which grow slowly and sustains for many human months or until the human end of life). Human acute diseases such as influenza virus and the disease symptoms include fever. The role of fibroblast growth factor 23 in kidney damage is investigated by metabolome analysis. The fibroblast growth factor controls blood phosphate levels through increasing renal phosphate excretion and declining vitamin D formation. The acute kidney damage increases fibroblast growth factor level, which increases the circulating glycerol-3-phosphate [45]. Metabolome analysis focuses on autophagy (lysosomal deprivation system), which possesses a protective role in kidney damage. The rubicon autophagy regulator increases the transfer of phospholipids from cells to lysosomes through increases in autophagy. The kidney epithelial cells and oleic acid increases fatty acids transport to mitochondria. The kidney epithelial cells increase triglyceride accumulation in hepatocytes which increases fatty acids movement from the kidney epithelial cells, and consequently, the metabolic syndrome occurs [46]. Metabolome helps researchers to realize the functional role of gastrointestinal tract microbial changes in human diseases such as inflammatory bowel disease. In inflammatory bowel disease in humans, amino acids are the major control key in this disease [47]. The metabolome is used to explore the existence of many biological states in the case of ischemic heart failure, which correlated with the variations in the lipid and hydrolase levels. Also, this study demonstrates that an activity-based protein tool is a suitable method to investigate the enzyme activity in human samples, leading to new drug and biomarker discovery in the future [48]. The inositol hexakisphosphate kinase 1 protein content in muscle is increased following lean meat intake in obese persons. This result refers to that inositol hexakisphosphate kinase 1 protein content in muscle leads to nutrient intake disturbances in skeletal muscle. Metabolome analysis proved that there are no differences in metabolites among obese, overweight, and lean persons [49]. Metabolome researches help in the understanding of many human diseases and consequently direct the scientists for clinical diagnostics. Metabolome results revealed a greater rise of immune cells to the human infected place to provide this place with the required energy supply and demand. The increase of $\mathrm{T}$ cells in human disease increases both glucose intake and glycolytic effect [50]. The metabolome analysis explores the human response to coronavirus infection. There are many circulating lipids, such as phosphatidylethanolamine, phosphatidylcholine, and phosphatidylcholine. These lipids serve as biomarkers of the coronavirus infection. There are increases in triglycerides and free fatty acids (oleic acid and arachidonic acid) associated with disease danger cases. The severity of the disease is accompanied by an increase in gluconeogenesis and the metabolism of porphyrins which lead to the development of the infection [51]. Metabolome analysis reveals the role of seizure protein 6 is related to many neurodegenerative diseases such as Alzheimer's disease. The seizure protein 6 controls cell surface localization and glycosylation of kainate receptors. So, help the researchers to understand the role of seizure protein 6 in neurologic and psychiatric 
diseases [52]. Metabolome helps scientists to investigate the mechanism involved in cardiovascular diseases. The results proved that the RNA interfering directing the natural anticoagulants antithrombin and protein $\mathrm{C}$ in human cardiovascular disease. In cardiovascular diseases, the proteins greatly affect the disease, especially the proteins related to erythrocyte function [53].

\subsection{Role of the metabolome in human chronic disease.}

Human chronic diseases remain for 1 year or more and need continuing medical precaution or limit activities of human dailydaily human life activities. Human chronic diseases include heart diseases, cancers, and diabetes that cause human death or disability. The phosphatase and tensin protein is overexpressed in human tumors. The chemotherapy is cancer inhibition depends on myeloid-cell phosphatase and tensin protein. This protein is important for chemotherapy to stimulate the pyrin domain containing 3 inflammasome and antitumor immunity [54]. Metabolome analysis is required to investigate the diminished fatty acid desaturation and lipid mediator difference inside the mesenteric adipose tissue, which leads to prolonged inflammation in Crohn's disease. The healing role of fatty acid desaturase- 2 is to lead to recovery of Crohn's disease [55]. Metabolome technique is applied in prolonged inflammation to investigate the role of tryptophan and kynurenine at the beginning of human depression. In human depression, the anthranilic acid level is increased while the tryptophan level is declined, but the kynurenine, kynurenic acid, 3-hydroxyanthranilic acid, and 3hydroxykynurenine levels are unchanged [56]. Liver fibrosis is the extreme accumulation of proteins in chronic liver disease. Ubiquitination is the process of chronic liver fibrosis pathophysiological process. This ubiquitin system has occurred in many human diseases. The metabolome technique proved that carbon tetrachloride caused liver fibrosis, which stimulates liver metabolome changes (sphingolipids and glycerophospholipids). The ubiquitination is included in the control of cell death, lipid metabolism, cell function, and DNA healing. So, the hepatic ubiquitome process leads to new therapeutic factors for the clinical managing of liver fibrosis [57]. The intestinal microbiota affects maturation and homeostasis of host immunity and sustains epithelial integrity with small metabolites. The immune cells obtain signals from microbiota which lead to the formation of a host-bacterial mutualism. The variations in the microbiota structure occur in patients with many disturbances such as inflammatory bowel disease [58]. The metabolome analysis proves the different role of erythrocyte hypoxic metabolic system in normal humans at high altitude. The patients with chronic kidney disease and sickle cell disease patients focus on the specific treatment to neutralize hypoxia among these groups [59]. The metabolome technique explores the Kai-Xin-San possesses antidepressant effect by controlling the signaling paths in lipid metabolism disturbances. The lipid metabolites are potential biomarkers of depression and specific places for Kai-Xin-San treatment of depression [60]. Metabolome analysis detected 79 metabolites, 27 of which were changed in the sciatic nerve. In the sciatic nerve, the glucose level is increased 2.6 times while glucose 6-phosphate is unchanged. The sciatic nerve declines the glycolytic activity and glycogen accumulation in skeletal muscle and leads to increased intramuscular glucose and polyol path intermediates [61]. 


\subsection{Role of the metabolome in human congenital disease.}

The congenital disturbance is a medical state that is occurred at or before human birth. This disease (also mentioned to birth defects) can be developed in the fetal growth stage or from the genetic transfer from the parents. Metabolome analysis is very useful to study the role of monosodium urate and calcium pyrophosphate crystals which induce metabolic redoing in the aerobic glycolysis path through the increase in glucose transporter 1 plasma membrane expression and glucose take on macrophages. Also, neutrophils taken from human synovial fluid during gout flare expressed glucose transporter 1 at their plasma membrane more regularly than neutrophils taken from the bloodstream [62]. The metabolome is a suitable tool to study nevoid basal cell carcinoma syndrome (Gorlin syndrome). This is a rare genetic disturbance that possesses obvious susceptibility to cerebral calcification, childhood medulloblastoma, basal cell carcinoma, maxillary keratocyst, and many soft and skeletal tissue developing irregularities. This disease contains the alteration of extracellular matrix, overexpression of growth factors, specific microRNAs, and basement membrane configuration, higher cytokines, and pro-angiogenic factors [63]. Metabolome study helps the researchers investigate the lysosomal storage disorder, including an increase of many substances such as mucopolysaccharides, sphingolipids, and oligosaccharides. The lysosome has a key role in the autophagy, where the combination of lysosome and autophagosome increases the breakdown of amino acids. The missing of lysosomal function leads to many indicators such as neurovisceral disturbances. This disease affects the human at birth and becomes symptomatic in childhood [64]. The metabolome study explores the deformity in cystathionine $\beta$-synthase deficit patients who have a decline in lysophosphatidylcholine and phosphatidylcholine, including an increase of polyunsaturated fatty acids and docosahexaenoic acid in lysophosphatidylethanolamine and phosphatidylethanol- amine.

The change in plasma S-adenosylhomocysteine and S-adenosylmethionine levels are correlated with phospholipid dysregulation. The docosahexaenoic acid plays an important role in thrombosis prevention. A decline of phosphatidylcholine contains docosahexaenoic acid in cystathionine $\beta$-synthase deficit patients. It helps the scientists investigate cardiovascular patients who possess homocystinuria [65]. The metabolome detects the changed small particles in nitric oxide exposure. The change in nitric oxide exposure contains the tricarboxylic acid cycle, nucleotide biosynthesis, and glutamine metabolism. The glutathione metabolites are increased with a decline in the glutathione precursor (glutamine). This process occurred with an increase in antioxidant enzymes. The glutamine deficiency is similar to that in human cytomegalovirus replication and mitochondrial respiration through nitric oxide exposure. So, nitric oxide restricts glutaminolysis through transporting of glutamine to glutathione. Nitric oxide interrupts many cellular processes and increasing of metabolic intermediates. Consequently, nitric oxide plays an important role in decreasing the human cytomegalovirus replication by many processes associated with viral replication inside the cell [66]. Metabolome technique helps to human diagnosis purpose. This technique investigates 268 distinguished metabolites in a group of 115 plasma samples. So, the metabolome method assists in applying metabolic diagnosis results, which improve diagnostic accuracy [67]. The metabolome analysis of oligodendrocyte and Schwann cell differentiation defects proves a decline in myelin basic protein level in the nervous system and development of defasciculated peripheral nerves. So, metabolome helps the researchers understand the hypomyelination (decline manufacture of myelin sheets by glial cells), including abnormal peripheral nerve transmission, hypotonia, 
cerebral hypomyelination, areflexia, and hypertrophic peripheral nerves [68]. Metabolome enables the accurate results of metabolites from cells, organs, tissues, or biofluids. Metabolome focus on the metabolism and the role of small molecule metabolites in many biological processes. These metabolites act as immune modulators, endogenous toxins, signaling molecules, and environmental sensors. So, metabolome improves the understanding of normal physiology and the pathophysiology of many human diseases through the metabolites affecting immune function, nutrient sensing, organ function, and gastrointestinal tract physiology [69].

\subsection{Role of the metabolome in human genetic disease.}

Genetic disease is a human health problem induced via one or more defects in the genome. This disease is induced by a mutation through a single gene (monogenic) or many genes (polygenic) or by a chromosomal aberration. Metabolome analysis explores the mutation of mitochondrial DNA that occurs in aging and human pathological conditions such as inherited errors of metabolism, tumors, and neurodegenerative diseases. The inhibitors of mitochondrial transcription damage DNA transcription mutation and cause inhibition of mutation of DNA expression [70]. Metabolome technique is a suitable method to apply in hormone receptor of breast cancer that induces breast cancer-associated deaths, which helps develop the therapeutic agents. In breast cancer, there is an increase in type I interferon secretion by malignant cells and consequently stimulation of immune effector cells. So, nicotinamide is a good agent applied in the prevention and treatment of hormone receptors of breast cancer [71]. Metabolome used to measure the expression of $\mathrm{G}$ protein receptor 55 in hepatic patients with nonalcoholic fatty liver disease. Metabolome study also the effects of 1$\alpha$-lysophosphatidylinositol and genetic disruption of $\mathrm{G}$ protein receptor 55 in animal and human cells. The results obtained showed that $1-\alpha$-lysophosphatidylinositol and $G$ protein receptor 55 are expressed in nonalcoholic steatohepatitis patients. The 1- $\alpha-$ lysophosphatidylinositol increases both liver stellate cells through stimulating $G$ protein receptor 55 and acetyl-coenzyme A carboxylase [72]. The metabolome approach investigates the short telomeres (major and essential key of telomere biology disturbances such as dyskeratosis congenital. In dyskeratosis, congenital, minor, and imbalance of nicotinamide adenine dinucleotide level is found. The intake of the nicotinamide adenine dinucleotide precursor (nicotinamide riboside) recovers the nicotinamide adenine dinucleotide homeostasis, which prevents telomere damage cell growth delay, and incomplete mitochondrial formation [73]. Metabolome enables researchers to study fatty acid synthesis in 2 ways; (1) in the cytoplasm (catalyzed by fatty acid synthase, FASN) and (2) in the mitochondria mutation of fatty acids (mtFAS). The mtFAS shows alternative metabolic effects such as reductive carboxylation. Also, the mtFAS damage stops the diversity of skeletal myoblasts in vitro. The mtFAS causes fatty acid synthesis and oxidation of carbon fuels [74]. Metabolome analysis shows that $13 \%$ of the lipids detected possess day and night cycles. These lipids are diacylglycerols and glycerophospholipids. The diacylglycerols are the biggest molecules. The diacylglycerols levels possess a day and night cycle with a peak at $4 \mathrm{AM}$ and a trough at $1 \mathrm{PM}$ [75]. Metabolome study reveals a relation is found between apolipoprotein E4 and Alzheimer's disease. apolipoprotein E4 affects many biological processes in the human brain, such as bioenergetics [76]. The metabolome is a suitable tool to investigate the gut microbiota effects on human health. Any changes in the microbiome metabolism affect human body homeostasis and correlated with many human neuropsychiatric and neurodegenerative disturbances such as Huntington's disease, autism spectrum disturbance, Parkinson's disease, Alzheimer's disease, 
bipolar disturbance, post-traumatic stress disturbance, depression, and schizophrenia. The gut microbiota plays an important role in oxidative stress, inflammation, and gut permeability [77]. Metabolome analysis reveals the primary cilium distracts many signaling paths and leads to the dysfunction of many human organs. The primary cilium is included in many biological processes such as tissue growth and matures tissues. The primary cilium induces kidney disease, so the primary cilium possesses an important role in kidney homeostasis. Metabolome is used to analysis of human urine. So, metabolome helps the scientist to understand the disease pathophysiology and in the detection of biomarkers [78].

\subsection{Role of the metabolome in incurable human disease.}

Incurable diseases are disturbances of infectious and non-infectious, autoimmune, genetic, neoplastic, or metabolic disease that have not any medical therapy. Incurable diseases contain many diseases such as late-stage of cardiac disease, AIDS, and tumors. Alzheimer's disease is an incurable neurodegenerative disease that is occurred higher in women. The metabolism is completely different in both males and females, so Alzheimer's disease is higher in females. In Alzheimer's disease, mitochondrial complex I-specific damage in cortical synaptic brain mitochondria in females but not males. In Alzheimer's disease, glial-increased and mitochondria in the hippocampus, cortex, and complex II-dependent respiration increased in females but not males. Metabolome technique reveals that systemic and brain metabolic reshifting from glucose to lipid metabolism in Alzheimer's disease in humans and animals. In Alzheimer's disease, there is an increase in glucose-6-phosphate. The glucose-6-phosphate is an inhibitor of hexokinase in the pentose phosphate path [79]. Gene therapy is a suitable factor for incurable diseases treatment. The gene possesses a major role in gene therapy techniques. So, the viral vectors act as a hyper-efficient method for gene therapy, such as adenoviral vectors which are applied in gene therapy in humans. The genes possess higher tendency to genes capability, efficiency, and expression. Consequently, adenoviral vectors must be controlled under good manufacturing practice methods before used in clinical trials [80]. Multiple myeloma is an incurable disease occurred within the bone marrow. The disease transports from premalignant stage (monoclonal gammopathy of undetermined significance) to intermediate stage (smoldering) to late-stage (multiple myeloma). The alterations of multiple myeloma cells to hypoxic situations in the bone marrow are responsible for multiple myeloma growth. Metabolome results of multiple myeloma lead to the detection of 6218 proteins. These proteins correlated with metabolic variations of the multiple myeloma cells, and this result will help in emerging therapeutic agents for multiple myeloma disease progression [81].

\section{Conclusion}

Metabolome mentioned to small molecules inside the biological sample. The metabolome includes both an endogenous metabolome and an exogenous metabolome. Human disease is an abnormal case that negatively affects the structure and function of one organism of the whole human body. Human disease is induced by external factors e.g., pathogens, or internal dysfunctions, e.g., hypersensitivity, immunity disorders, autoimmune disorders, immunodeficiency, and allergy. Humans' disease is accompanied by distress, pain, social problems, dysfunction, or death. There are 6 different types of human diseases: acquired disease, acute disease, chronic disease, congenital disease, genetic disease, an incurable 
disease. In conclusion, the metabolome plays an important role in the above-mentioned human diseases' therapeutic basis.

\section{Funding}

This review received no external funding.

\section{Acknowledgments}

This review has no acknowledgment.

\section{Conflicts of Interest}

The authors declare no conflict of interest.

\section{References}

1. Oliver, S.G.; Winson, M.K.; Kell, D.B.; Baganz, F. Systematic functional analysis of the yeast genome. Trends Biotechnol 1998, 16, 373-378, https://doi.org/10.1016/S0167-7799(98)01214-1.

2. Wishart, D.S. Current progress in computational metabolomics. Brief Bioinform 2007, 8, 279-293, https://doi.org/10.1093/bib/bbm030.

3. Nordström, A.; O'Maille, G.; Qin, C.; Siuzdak, G. Nonlinear data alignment for UPLC-MS and HPLC-MS based metabolomics: quantitative analysis of endogenous and exogenous metabolites in human serum. Anal Chem 2006, 78, 3289-3295, https://doi.org/10.1021/ac060245f.

4. Tweeddale, H.; Notley-McRobb, L.; Ferenci, T. Effect of slow growth on metabolism of Escherichia coli, as revealed by global metabolite pool ("metabolome") analysis. J Bacteriol 1998, 180, 5109-5116.

5. Koriem, K.M.M. A lipidomic concept in infectious diseases. Asian Pac J Trop Biomed 2017, 7, 265-274, https://doi.org/10.1016/j.apjtb.2016.12.010.

6. Koriem, K.M.M. Protective effect of natural products and hormones in colon cancer using metabolome: A physiological overview. Asian Pac J Trop Biomed 2017, 7, 957-966, https://doi.org/10.1016/j.apjtb.2017.09.002.

7. Koriem, K.M.M. Proteomic approach in human health and disease: Preventive and cure studies. Asian Pac J Trop Biomed 2018, 8, 226-236, https://doi.org/10.4103/2221-1691.231285.

8. Koriem, K.M.M. Lipidome is lipids regulator in gastrointestinal tract and it is a life collar in COVID-19: A review. World J Gastroenterol 2021, 27, 37-54, https://doi.org/10.3748/wjg.v27.i1.37.

9. Sharma, R.; Ramanathan, A. The aging metabolome-biomarkers to hub metabolites. Proteomics 2020, 20, https://doi.org/10.1002/pmic.201800407.

10. Bar, N.; Korem, T.; Weissbrod, O.; Zeevi, D.; Rothschild, D.; Leviatan, S.; Kosower, N.; Lotan-Pompan, M.; Weinberger, A.; Le Roy, C.I.; Menni, C.; Visconti, A.; Falchi, M.; Spector, T.D.; IMI DIRECT consortium, Adamski, J.; Franks, P.W.; Pedersen, O.; Segal, E. A reference map of potential determinants for the human serum metabolome. Nature 2020, 588, 135-140, https://doi.org/10.1038/s41586-020-2896-2.

11. Van Treuren, W.; Dodd, D. Microbial Contribution to the Human Metabolome: Implications for Health and Disease. Аnпи Rev Pathol 2020, 15, 345-369, https://doi.org/10.1146/annurev-pathol-020117-043559.

12. Bittinger, K.; Zhao, C.; Li, Y.; Ford, E.; Friedman, E.S.; Ni, J.; Kulkarni, C.V.; Cai, J.; Tian, Y.; Liu, Q.; Patterson, A.D.; Sarkar, D.; Chan, S.H.J.; Maranas, C.; Saha-Shah, A.; Lund, P.; Garcia, B.A.; Mattei, L.M.; Gerber, J.S.; Elovitz, M.A.; Kelly, A.; DeRusso, P.; Kim, D.; Hofstaedter, C.E.; Goulian, M.; Li, H.; Bushman, F.D.; Zemel, B.S.; Wu, G.D. Bacterial colonization reprograms the neonatal gut metabolome. Nat Microbiol 2020, 5, 838-847, https://doi.org/10.1038/s41564-020-0694-0.

13. Huo, Z.; Yu, L.; Yang, J.; Zhu, Y.; Bennett, D.A.; Zhao, J. Brain and blood metabolome for Alzheimer's dementia: findings from a targeted metabolomics analysis. Neurobiol Aging 2020, 86, 123-133, https://doi.org/10.1016/j.neurobiolaging.2019.10.014.

14. Lawler, K.; Huang-Doran, I.; Sonoyama, T.; Collet, T.H.; Keogh, J.M.; Henning, E.; O'Rahilly, S.; Bottolo, L.; Farooqi, I.S. Leptin-mediated changes in the human metabolome. J Clin Endocrinol Metab 2020, 105, 2541-2552, https://doi.org/10.1210/clinem/dgaa251.

15. Malik, D.M.; Paschos, G.K.; Sehgal, A.; Weljie, A.M. Circadian and Sleep Metabolomics Across Species. J Mol Biol 2020, 432, 3578-3610, https://doi.org/10.1016/j.jmb.2020.04.027.

16. Trifonova, O.P.; Balashova, E.E.; Maslov, D.L.; Grigoriev, A.I.; Lisitsa, A.V.; Ponomarenko, E.A.; Archakov, A.I. Blood metabolome analysis for creating a digital image of a healthy person. Biomed Khim 2020, 66, 216-223, https://doi.org/10.18097/PBMC20206603216.

17. Vairakkani, R.; Fernando, M.E.; Raj, T.Y. Metabolome and microbiome in kidney diseases. Saudi J Kidney Dis Transpl 2020, 31, 1-9, https://doi.org/10.4103/1319-2442.279927. 
18. Crestani, E.; Harb, H.; Charbonnier, L.M.; Leirer, J.; Motsinger-Reif, A.; Rachid, R.; Phipatanakul, W.; Kaddurah-Daouk, R.; Chatila, T.A. Untargeted metabolomic profiling identifies disease-specific signatures in food allergy and asthma. $J$ Allergy Clin Immunol 2020, 145, 897-906, https://doi.org/10.1016/j.jaci.2019.10.014.

19. Nguyen, T.; Sedghi, L.; Ganther, S.; Malone, E.; Kamarajan, P.; Kapila, Y.L. Host-microbe interactions: Profiles in the transcriptome, the proteome, and the metabolome. Periodontol 2000 2020, 82, 115-128, https://doi.org/10.1111/prd.12316.

20. Li, D.; Ma, B.; Xu, X.; Chen, G.; Li, T.; He, N. MMHub, a database for the mulberry metabolome. Database (Oxford) 2020, 2020, https://doi.org/10.1093/database/baaa011.

21. Kosmacz, M.; Sokołowska, E.M.; Bouzaa, S.; Skirycz, A. Towards a functional understanding of the plant metabolome. Curr Opin Plant Biol 2020, 55, 47-51, https://doi.org/10.1016/j.pbi.2020.02.005.

22. Zhang, Z.; Yi, P.; Yang, J.; Huang, J.; Xu, P.; Hu, M.; Zhang, C.; Wang, B.; Peng, W. Integrated network pharmacology analysis and serum metabolomics to reveal the cognitive improvement effect of Bushen Tiansui formula on Alzheimer's disease. $J$ Ethnopharmacol 2020, 249, https://doi.org/10.1016/j.jep.2019.112371.

23. Hunsberger, H.C.; Greenwood, B.P.; Tolstikov, V.; Narain, N.R.; Kiebish, M.A.; Denny, C.A. Divergence in the metabolome between natural aging and Alzheimer's disease. Sci Rep 2020, 10, https://doi.org/10.1038/s41598-020-68739-z.

24. Feng, Z.; Ding, C.; Li, W.; Wang, D.; Cui, D. Applications of metabolomics in the research of soybean plant under abiotic stress. Food Chem 2020, 310, https://doi.org/10.1016/j.foodchem.2019.125914.

25. Bonifácio, V.D.B. Ovarian cancer biomarkers: Moving forward in early detection. Adv Exp Med Biol 2020, 1219, 355-363, https://doi.org/10.1007/978-3-030-34025-4_18.

26. Rodríguez-Gómez, J.A.; Kavanagh, E.; Engskog-Vlachos, P.; Engskog, M.K.R.; Herrera, A.J.; EspinosaOliva, A.M.; Joseph, B.; Hajji, N.; Venero, J.L.; Burguillos, M.A. Microglia: agents of the CNS proInflammatory response. Cells 2020, 9, https://doi.org/10.3390/cells9071717.

27. Piersigilli, F.; Van Grambezen, B.; Hocq, C.; Danhaive, O. Nutrients and microbiota in lung diseases of prematurity: The placenta-gut-lung triangle. Nutrients 2020, 12, https://doi.org/10.3390/nu12020469.

28. Sinha, S.R.; Haileselassie, Y.; Nguyen, L.P.; Tropini, C.; Wang, M.; Becker, L.S.; Sim, D.; Jarr, K.; Spear, E.T.; Singh, G.; Namkoong, H.; Bittinger, K.; Fischbach, M.A.; Sonnenburg, J.L.; Habtezion, A. Dysbiosisinduced secondary bile acid deficiency promotes intestinal inflammation. Cell Host Microbe 2020, 27, 659670, https://doi.org/10.1016/j.chom.2020.01.021.

29. Murgia, F.; Atzori, L.; Carboni, E.; Santoru, M.L.; Hendren, A.; Pisanu, A.; Caboni, P.; Boi, L.; Fusco, G.; Carta, A.R. Metabolomics fingerprint induced by the intranigral inoculation of exogenous human alphasynuclein oligomers in a rat model of parkinson's disease. Int $J$ Mol Sci 2020, 21, https://doi.org/10.3390/ijms21186745.

30. Langan, D.; Rose, N.R.; Moudgil, K.D. Common innate pathways to autoimmune disease. Clin Immunol 2020, 212, https://doi.org/10.1016/j.clim.2020.108361.

31. Li, A.M.; Ducker, G.S.; Li, Y.; Seoane, J.A.; Xiao, Y.; Melemenidis, S.; Zhou, Y.; Liu, L.; Vanharanta, S.; Graves, E.E.; Rankin, E.B.; Curtis, C.; Massagué, J.; Rabinowitz, J.D.; Thompson, C.B.; Ye, J. Metabolic profiling reveals a dependency of human metastatic breast cancer on mitochondrial serine and one-carbon unit metabolism. Mol Cancer Res 2020, 18, 599-611, https://doi.org/10.1158/1541-7786.MCR-19-0606.

32. Bardanzellu, F.; Peroni, D.G.; Fanos, V. Human breast milk: Bioactive components, from stem cells to health outcomes. Curr Nutr Rep 2020, 9, 1-13, https://doi.org/10.1007/s13668-020-00303-7.

33. Botticelli, A.; Vernocchi, P.; Marini, F.; Quagliariello, A.; Cerbelli, B.; Reddel, S.; Del Chierico, F.; Di Pietro, F.; Giusti, R.; Tomassini, A.; Giampaoli, O.; Miccheli, A.; Zizzari, I.G.; Nuti, M.; Putignani, L.; Marchetti, P. Gut metabolomics profiling of non-small cell lung cancer (NSCLC) patients under immunotherapy treatment. J Transl Med 2020, 18, https://doi.org/10.1186/s12967-020-02231-0.

34. Hu, X.; Li, S.; Cirillo, P.; Krigbaum, N.; Tran, V.; Ishikawa, T.; La Merrill, M.A.; Jones, D.P.; Cohn, B. Metabolome wide association study of serum DDT and DDE in pregnancy and early postpartum. Reprod Toxicol 2020, 92, 129-137, https://doi.org/10.1016/j.reprotox.2019.05.059.

35. Nugent, A.A.; Lin, K.; van Lengerich, B.; Lianoglou, S.; Przybyla, L.; Davis, S.S.; Llapashtica, C.; Wang, J.; Kim, D.J.; Xia, D.; Lucas, A,; Baskaran, S.; Haddick, P.C.G.; Lenser, M.; Earr, T.K.; Shi, J.; Dugas, J.C.; Andreone, B.J.; Logan, T.; Solanoy, H.O.; Chen, H.; Srivastava, A.; Poda, S.B.; Sanchez, P.E.; Watts, R.J.; Sandmann, T.; Astarita, G.; Lewcock, J.W.; Monroe, K.M.; Di Paolo, G. TREM2 Regulates Microglial Cholesterol Metabolism upon Chronic Phagocytic Challenge. Neuron 2020, 105, 837-854.e9, https://doi.org/10.1016/j.neuron.2019.12.007.

36. Ismail, I.T.; Fiehn, O.; Elfert, A.; Helal, M.; Salama, I.; El-Said, H. Sugar Alcohols Have a key role in pathogenesis of chronic liver disease and hepatocellular carcinoma in whole blood and liver tissues. Cancers (Basel) 2020, 12, https://doi.org/10.3390/cancers12020484.

37. Guillon, A.; Arafa, E.I.; Barker, K.A.; Belkina, A.C.; Martin, I.; Shenoy, A.T.; Wooten, A.K.; Lyon De Ana, C.; Dai, A.; Labadorf, A.; Hernandez Escalante, J.; Dooms, H.; Blasco, H.; Traber, K.E.; Jones, M.R.; Quinton, L.J.; Mizgerd, J.P. Pneumonia recovery reprograms the alveolar macrophage pool. JCI Insight 2020, 5, https://doi.org/10.1172/jci.insight.133042. 
38. Wang, F.; Meng, J.; Zhang, L.; Roy, S. Opioid use potentiates the virulence of hospital-acquired infection, increases systemic bacterial dissemination and exacerbates gut dysbiosis in a murine model of Citrobacter rodentium infection. Gut Microbes 2020, 11, 172-190, https://doi.org/10.1080/19490976.2019.1629237.

39. Cerboni, S.; Gehrmann, U.; Preite, S.; Mitra, S. Cytokine-regulated Th17 plasticity in human health and diseases. Immunology 2020, https://doi.org/10.1111/imm.13280.

40. Rubio-Jurado, B.; Sosa-Quintero, L.S.; Carrasco-Martinez, I.L.; Norato-Delgado, A.; Garcia-Luna, E.; Guzmán-Silahua, S.; Riebeling-Navarro, C.; Nava-Zavala, A.H. New biomarkers in non-Hodgkin lymphoma and acute leukemias. Adv Clin Chem 2020, 96, 19-53, https://doi.org/10.1016/bs.acc.2019.11.002.

41. Gojon, G.; Morales, G.A. SG1002 and catenated divalent organic sulfur compounds as promising hydrogen sulfide prodrugs. Antioxid Redox Signal 2020, 33, 1010-1045, https://doi.org/10.1089/ars.2020.8060.

42. Sieow, B.F.; Nurminen, T.J.; Ling, H.; Chang, M.W. Meta-omics- and metabolic modeling-assisted deciphering of human microbiota metabolism. Biotechnol J 2019, 14, https://doi.org/10.1002/biot.201800445.

43. Ivanova, O.; Richards, L.B.; Vijverberg, S.J.; Neerincx, A.H.; Sinha, A.; Sterk, P.J.; Maitland-van der Zee, A.H. What did we learn from multiple omics studies in asthma?. Allergy 2019, 74, 2129-2145, https://doi.org/10.1111/all.13833.

44. Sottero, B.; Rossin, D.; Staurenghi, E.; Gamba, P.; Poli, G.; Testa, G. Omics analysis of oxysterols to better understand their pathophysiological role. Free Radic Biol Med 2019, 144, 55-71, https://doi.org/10.1016/j.freeradbiomed.2019.05.026.

45. Simic, P.; Kim, W.; Zhou, W.; Pierce, K.A.; Chang, W.; Sykes, D.B.; Aziz, N.B.; Elmariah, S.; Ngo, D.; Pajevic, P.D.; Govea, N.; Kestenbaum, B.R.; de Boer, I.H.; Cheng, Z.; Christov, M.; Chun, J.; Leaf, D.E.; Waikar, S.S.; Tager, A.M.; Gerszten, R.E.; Thadhani, R.I.; Clish, C.B.; Jüppner, H.; Wein, M.N.; Rhee, E.P. Glycerol-3-phosphate is an FGF23 regulator derived from the injured kidney. J Clin Invest 2020, 130, 15131526, https://doi.org/10.1172/JCI131190.

46. Matsuda, J.; Takahashi, A.; Takabatake, Y.; Sakai, S.; Minami, S.; Yamamoto, T.; Fujimura, R.; NambaHamano, T.; Yonishi, H.; Nakamura, J.; Kimura, T.; Kaimori, J.Y.; Matsui, I.; Takahashi, M.; Nakao, M.; Izumi, Y.; Bamba, T.; Matsusaka, T.; Niimura, F.; Yanagita, M.; Yoshimori, T.; Isaka, Y. Metabolic effects of RUBCN/Rubicon deficiency in kidney proximal tubular epithelial cells. Autophagy 2020, 16, 1889-1904, https://doi.org/10.1080/15548627.2020.1712107.

47. Knudsen, L.A.; Desdorf, R.; Möller, S.; Sørensen, S.B.; Hansen, A.K.; Andersen, V. Translational potential of metabolomics on animal models of inflammatory bowel disease-A systematic critical review. Int J Mol Sci 2020, 21, https://doi.org/10.3390/ijms21113856.

48. $\quad$ van Esbroeck, A.C.M.; Varga, Z.V.; Di, X.; van Rooden, E.J.; Tóth, V.E.; Onódi, Z.; Kuśmierczyk, M.; Leszek, P.; Ferdinandy, P.; Hankemeier, T.; van der Stelt, M.; Pacher, P. Activity-based protein profiling of the human failing ischemic heart reveals alterations in hydrolase activities involving the endocannabinoid system. Pharmacol Res 2020, 151, https://doi.org/10.1016/j.phrs.2019.104578.

49. Barclay, R.D.; Beals, J.W.; Drnevich, J.; Imai, B.S.; Yau, P.M.; Ulanov, A.V.; Tillin, N.A.; Villegas-Montes, M.; Paluska, S.A.; Watt, P.W.; De Lisio, M.; Burd, N.A.; Mackenzie, R.W. Ingestion of lean meat elevates muscle inositol hexakisphosphate kinase 1 protein content independent of a distinct post-prandial circulating proteome in young adults with obesity. Metabolism 2020, 102, https://doi.org/10.1016/j.metabol.2019.153996.

50. Kumari, R.; Palaniyandi, S.; Hildebrandt, G.C. Metabolic Reprogramming-A New Era How to Prevent and Treat Graft Versus Host Disease After Allogeneic Hematopoietic Stem Cell Transplantation Has Begun. Front Pharmacol 2020, 11, https://doi.org/10.3389/fphar.2020.588449.

51. Barberis, E.; Timo, S.; Amede, E.; Vanella, V.V.; Puricelli, C.; Cappellano, G.; Raineri, D.; Cittone, M.G.; Rizzi, E.; Pedrinelli, A.R.; Vassia, V,; Casciaro, F,G,; Priora, S.; Nerici, I.; Galbiati, A.; Hayden, E.; Falasca, M.; Vaschetto, R.; Sainaghi, P.P.; Dianzani, U.; Rolla, R.; Chiocchetti, A.; Baldanzi, G.; Marengo, E.; Manfredi, M. Large-scale plasma analysis revealed new mechanisms and molecules associated with the Host Response to SARS-CoV-2. Int J Mol Sci 2020, 21, https://doi.org/10.3390/ijms21228623 .

52. Pigoni, M.; Hsia, H.E.; Hartmann, J.; Rudan Njavro, J.; Shmueli, M.D.; Müller, S.A.; Güner, G.; Tüshaus, J.; Kuhn, P.H.; Kumar, R.; Gao, P.; Tran, M.L.; Ramazanov, B.; Blank, B.; Hipgrave Ederveen, A.L.; Von Blume, J.; Mulle, C.; Gunnersen, J.M.; Wuhrer, M.; Rammes, G.; Busche, M.A.; Koeglsperger, T.; Lichtenthaler, S.F. Seizure protein 6 controls glycosylation and trafficking of kainate receptor subunits GluK2 and GluK3. EMBO J 2020, 39, https://doi.org/10.15252/embj.2019103457.

53. Tilburg, J.; Michaud, S.A.; Maracle, C.X.; Versteeg, H.H.; Borchers, C.H.; van Vlijmen, B.J.M.; Mohammed, Y. Plasma protein signatures of a murine venous thrombosis model and Slc44a2 knockout mice using quantitative-targeted proteomics. Thromb Haemost 2020, 120, 423-436, https://doi.org/10.1055/s0040-1702229.

54. Huang, Y.; Wang, H.; Hao, Y.; Lin, H.; Dong, M.; Ye, J.; Song, L.; Wang, Y.; Li, Q.; Shan, B.; Jiang, Y.; Li, H.; Shao, Z.; Kroemer, G.; Zhang, H.; Bai, L.; Jin, T.; Wang, C.; Ma, Y.; Cai, Y.; Ding, C.; Liu, S.; Pan, Y.; Jiang, W.; Zhou, R. Myeloid PTEN promotes chemotherapy-induced NLRP3-inflammasome activation and antitumour immunity. Nat Cell Biol 2020, 22, 716-727, https://doi.org/10.1038/s41556-020-0510-3. 
55. Liu, R.; Qiao, S.; Shen, W.; Liu, Y.; Lu, Y.; Liangyu, H.; Guo, Z.; Gong, J.; Shui, G.; Li, Y.; Zhu, W. Disturbance of fatty acid desaturation mediated by FADS2 in mesenteric adipocytes contributes to chronic inflammation of Crohn's disease. J Crohns Colitis 2020, 14, 1581-1599, https://doi.org/10.1093/eccojcc/jjaa086.

56. Sakurai, M.; Yamamoto, Y.; Kanayama, N.; Hasegawa, M.; Mouri, A.; Takemura, M.; Matsunami, H.; Miyauchi, T.; Tokura, T.; Kimura, H.; Ito, M.; Umemura, E.; Boku, A.S.; Nagashima, W.; Tonoike, T.; Kurita, K.; Ozaki, N.; Nabeshima, T.; Saito, K. Serum metabolic profiles of the tryptophan-kynurenine pathway in the high risk subjects of major depressive disorder. Sci Rep 2020, 10, https://doi.org/10.1038/s41598-020-58806-w.

57. Mercado-Gómez, M.; Lopitz-Otsoa, F.; Azkargorta, M.; Serrano-Maciá, M.; Lachiondo-Ortega, S.; Goikoetxea-Usandizaga, N.; Rodríguez-Agudo, R.; Fernández-Ramos, D.; Bizkarguenaga, M.; Juan, V.G.; Lectez, B.; Aloria, K.; Arizmendi, J.M.; Simon, J.; Alonso, C.; Lozano, J.J.; Avila, M.A.; Banales, J.M.; Marin, J.J.G.; Beraza, N.; Mato, J.M.; Elortza, F.; Barrio, R.; Sutherland, J.D.; Mayor, U.; Martínez-Chantar, M.L.; Delgado, T.C. Multi-omics integration highlights the role of ubiquitination in $\mathrm{CCl}_{4}$-induced liver fibrosis. Int J Mol Sci 2020, 21, https://doi.org/10.3390/ijms21239043.

58. Kayama, H.; Takeda, K. Manipulation of epithelial integrity and mucosal immunity by host and microbiotaderived metabolites. Eur J Immunol 2020, 50, 921-931, https://doi.org/10.1002/eji.201948478.

59. D'Alessandro, A.; Xia, Y. Erythrocyte adaptive metabolic reprogramming under physiological and pathological hypoxia. Curr Opin Hematol 2020, 27, 155-162, https://doi.org/10.1097/MOH.0000000000000574.

60. Zhou, X.; Wang, J.; Lu, Y.; Chen, C.; Hu, Y.; Liu, P.; Dong, X. Anti-depressive effects of Kai-Xin-San on lipid metabolism in depressed patients and CUMS rats using metabolomic analysis. J Ethnopharmacol 2020, 252, https://doi.org/10.1016/j.jep.2020.112615.

61. Langer, H.T.; Afzal, S.; Kempa, S.; Spuler, S. Nerve damage induced skeletal muscle atrophy is associated with increased accumulation of intramuscular glucose and polyol pathway intermediates. Sci Rep 2020, 10, https://doi.org/10.1038/s41598-020-58213-1.

62. Renaudin, F.; Orliaguet, L.; Castelli, F.; Fenaille, F.; Prignon, A.; Alzaid, F.; Combes, C.; Delvaux, A.; Adimy, Y.; Cohen-Solal, M.; Richette, P.; Bardin, T.; Riveline, J.P.; Venteclef, N.; Lioté, F.; CampilloGimenez, L.; Ea, H.K. Gout and pseudo-gout-related crystals promote GLUT1-mediated glycolysis that governs NLRP3 and interleukin-1 $\beta$ activation on macrophages. Ann Rheum Dis 2020, 79, 1506-1514, https://doi.org/10.1136/annrheumdis-2020-217342.

63. Bellei, B.; Caputo, S.; Carbone, A.; Silipo, V.; Papaccio, F.; Picardo, M.; Eibenschutz, L. The role of dermal fibroblasts in nevoid basal cell carcinoma syndrome patients: An overview. Int J Mol Sci 2020, 21, https://doi.org/10.3390/ijms21030720.

64. Mashima, R.; Okuyama, T.; Ohira, M. Biomarkers for Lysosomal Storage Disorders with an Emphasis on Mass Spectrometry. Int J Mol Sci 2020, 21, https://doi.org/10.3390/ijms21082704.

65. Di Minno, A.; Anesi, A.; Chiesa, M.; Cirillo, F.; Colombo, G.I.; Orsini, R.C.; Capasso, F.; Morisco, F.; Fiorelli, S.; Eligini, S.; Cavalca, V.; Tremoli, E.; Porro, B.; Di Minno, M.N.D. Plasma phospholipid dysregulation in patients with cystathionine- $\beta$ synthase deficiency. Nutr Metab Cardiovasc Dis 2020, 30, 2286-2295, https://doi.org/10.1016/j.numecd.2020.07.019.

66. Mokry, R.L.; Schumacher, M.L.; Hogg, N.; Terhune, S.S. Nitric oxide circumvents virus-mediated metabolic regulation during human ctomegalovirus infection. mBio 2020, 11, e02630-20, https://doi.org/10.1128/mBio.02630-20.

67. Haijes, H,A.; van der Ham, M.; Prinsen, H.C.M.T.; Broeks, M.H.; van Hasselt, P.M.; de Sain-van der Velden, M.G.M.; Verhoeven-Duif, N.M.; Jans, J.J.M. Untargeted metabolomics for metabolic diagnostic screening with automated data interpretation using a knowledge-based algorithm. Int J Mol Sci 2020, 21, https://doi.org/10.3390/ijms21030979.

68. Blanco-Sánchez, B.; Clément, A.; Stednitz, S.J.; Kyle, J.; Peirce, J.L.; McFadden, M.; Wegner, J.; Phillips, J.B.; Macnamara, E.; Huang, Y.; Adams, D.R.; Toro, C.; Gahl, W.A.; Malicdan, M.C.V.; Tifft, C.J.; Zink, E.M.; Bloodsworth, K.J.; Stratton, K.G. Undiagnosed Diseases Network, Koeller, D.M.; Metz, T.O.; Washbourne, P.; Westerfield, M. yippee like 3 (ypel3) is a novel gene required for myelinating and perineurial glia development. PLoS Genet 2020, 16, https://doi.org/10.1371/journal.pgen.1008841.

69. Wishart, D.S. Metabolomics for investigating physiological and pathophysiolog-ical processes. Physiol Rev 2019, 99, 1819-1875, https://doi.org/10.1152/physrev.00035.2018.

70. Bonekamp, N.A.; Peter, B.; Hillen, H.S.; Felser, A.; Bergbrede, T.; Choidas, A.; Horn, M.; Unger, A.; Di Lucrezia, R.; Atanassov, I.; Li, X.; Koch, U.; Menninger, S.; Boros, J.; Habenberger, P.; Giavalisco, P.; Cramer, P.; Denzel, M.S.; Nussbaumer, P.; Klebl, B.; Falkenberg, M.; Gustafsson, C.M.; Larsson, N.G. Small-molecule inhibitors of human mitochondrial DNA transcription. Nature 2020, 588, 712-716, https://doi.org/10.1038/s41586-020-03048-z.

71. Buqué, A.; Bloy, N.; Perez-Lanzón, M.; Iribarren, K.; Humeau, J.; Pol, J.G.; Levesque, S.; Mondragon, L.; Yamazaki, T.; Sato, A.; Aranda, F.; Durand, S.; Boissonnas, A.; Fucikova, J.; Senovilla, L.; Enot, D.; Hensler, M.; Kremer, M.; Stoll, G.; Hu, Y.; Massa, C.; Formenti, S.C.; Seliger, B.; Elemento, O.; Spisek, R.; André, F.; Zitvogel, L.; Delaloge, S.; Kroemer, G.; Galluzzi, L. Immunoprophylactic and immunotherapeutic 
control of hormone receptor-positive breast cancer. Nat Commun 2020, 11, https://doi.org/10.1038/s41467020-17644-0.

72. Fondevila, M.F.; Fernandez, U.; Gonzalez-Rellan, M.J.; Da Silva Lima, N.; Buque, X.; Gonzalez-Rodriguez, A.; Alonso, C.; Iruarrizaga-Lejarreta, M.; Delgado, T.C.; Varela-Rey, M.; Senra, A.; Garcia-Outeiral, V.; Novoa, E.; Iglesias, C.; Porteiro, B.; Beiroa, D.; Folgueira, C.; Tojo, M.; Torres, J.L.; Hernández-Cosido, L.; Blanco, Ó.; Arab, J.P.; Barrera, F.; Guallar, D.; Fidalgo, M.; López, M.; Dieguez, C.; Marcos, M.; Martinez-Chantar, M.L.; Arrese, M.; Garcia-Monzon, C.; Mato, J.M.; Aspichueta, P.; Nogueiras, R. The L$\alpha$-Lysophosphatidylinositol/G Protein-Coupled Receptor 55 System Induces the Development of Nonalcoholic Steatosis and Steatohepatitis. Hepatology 2020, https://doi.org/10.1002/hep.31290.

73. Sun, C.; Wang, K.; Stock, A.J.; Gong, Y.; Demarest, T.G.; Yang, B.; Giri, N.; Harrington, L.; Alter, B.P.; Savage, S.A.; Bohr, V.A.; Liu, Y. Re-equilibration of imbalanced NAD metabolism ameliorates the impact of telomere dysfunction. EMBO J 2020,39, https://doi.org/10.15252/embj.2019103420.

74. Nowinski, S.M.; Solmonson, A.; Rusin, S.F.; Maschek, J.A.; Bensard, C.L.; Fogarty, S.; Jeong, M.Y.; Lettlova, S.; Berg, J.A.; Morgan, J.T.; Ouyang, Y.; Naylor, B.C.; Paulo, J.A.; Funai, K.; Cox, J.E.; Gygi, S.P.; Winge, D.R.; DeBerardinis, R.J.; Rutter, J. Mitochondrial fatty acid synthesis coordinates oxidative metabolism in mammalian mitochondria. Elife 2020, 9, https://doi.org/10.7554/eLife.58041.

75. Held, N.M.; Wefers, J.; van Weeghel, M.; Daemen, S.; Hansen, J.; Vaz, F.M.; van Moorsel, D.; Hesselink, M.K.C.; Houtkooper, R.H.; Schrauwen, P. Skeletal muscle in healthy humans exhibits a day-night rhythm in lipid metabolism. Mol Metab 2020, 37, https://doi.org/10.1016/j.molmet.2020.100989.

76. Area-Gomez, E.; Larrea, D.; Pera, M.; Agrawal, R.R.; Guilfoyle, D.N.; Pirhaji, L.; Shannon, K.; Arain, H.A.; Ashok, A.; Chen, Q.; Dillman, A.A.; Figueroa, H.Y.; Cookson, M.R.; Gross, S.S.; Fraenkel, E.; Duff, K.E.; Nuriel, T. APOE4 is associated with differential regional vulnerability to bioenergetic deficits in aged APOE mice. Sci Rep 2020, 10, https://doi.org/10.1038/s41598-020-61142-8.

77. Konjevod, M.; Nikolac Perkovic, M.; Sáiz, J.; Svob Strac, D.; Barbas, C.; Rojo, D. Metabolomics analysis of microbiota-gut-brain axis in neurodegenerative and psychiatric diseases. J Pharm Biomed Anal 2021, 194, https://doi.org/10.1016/j.jpba.2020.113681.

78. Zacchia, M.; Marchese, E.; Trani, E.M.; Caterino, M.; Capolongo, G.; Perna, A.; Ruoppolo, M.; Capasso, G. Proteomics and metabolomics studies exploring the pathophysiology of renal dysfunction in autosomal dominant polycystic kidney disease and other ciliopathies. Nephrol Dial Transplant 2020, 35, 1853-1861, https://doi.org/10.1093/ndt/gfz121.

79. Demarest, T.G.; Varma, V.R.; Estrada, D.; Babbar, M.; Basu, S.; Mahajan, U.V.; Moaddel, R.; Croteau, D.L.; Thambisetty, M.; Mattson, M.P.; Bohr, V.A. Biological sex and DNA repair deficiency drive Alzheimer's disease via systemic metabolic remodeling and brain mitochondrial dysfunction. Acta Neuropathol 2020, 140, 25-47, https://doi.org/10.1007/s00401-020-02152-8.

80. Arjmand, B.; Alavi-Moghadam, S.; Payab, M.; Goodarzi, P.; Sheikh Hosseini, M.; Tayanloo-Beik, A.; Rezaei-Tavirani, M.; Larijani, B. GMP-compliant adenoviral vectors for gene therapy. Methods Mol Biol 2020, 237-350, https://doi.org/10.1007/7651_2020_284.

81. Janker, L.; Mayer, R.L.; Bileck, A.; Kreutz, D.; Mader, J.C.; Utpatel, K.; Heudobler, D.; Agis, H.; Gerner, C.; Slany, A. Metabolic, anti-apoptotic and immune evasion strategies of primary human myeloma cells indicate adaptations to hypoxia. Mol Cell Proteomics 2019, 18, 936-953, https://doi.org/10.1074/mcp.RA119.001390. 